\title{
Comparative efficacy of fluconazole and liposomal amphotericin B in diabetic mice infected with an azole resistant or sensitive Candida albicans
}

\author{
Shirleen Simargi, Jon Olson, Jill Adler-Moore \\ California State Polytechnic University, Pomona, USA (Email: sisimargi@cpp.edu)
}

\begin{abstract}
Objective: Diabetes compromises blood circulation and immune responses, as well as major metabolic pathways, which can lead to increased susceptibility to infection. In the present study, we examined this possibility using diabetic versus non-diabetic mice with systemic candidiasis caused by a Candida albicans azole sensitive(C.alb-S) or azole resistant(C.alb-R) strain. Infected mice were treated with fluconazole (Flu) or liposomal amphotericin B (AmBisome $₫, A m B i)$. Methods: ICR male mice (3-4 weeks old) were maintained on a high $(60 \%)$ fat diet for 4 weeks. Diabetes was then chemically induced by one intraperitoneal nicotinamide $(60 \mathrm{mg} / \mathrm{kg}$ ) and streptozotocin $(100 \mathrm{mg} / \mathrm{kg})$ treatment; by day 7-14 mean blood glucose levels were $\geq 200 \mathrm{mg} / \mathrm{dL}$ Diabetic mice ( $\mathrm{n}=9-14 / \mathrm{gp}$ ) and nondiabetic mice ( $n=14-16 / g p)$ were challenged intravenously (IV) with C.alb-R (ATCC 62342) (diabetic: 3.5x10ex6 cells/mouse; non-diabetic: 1.4x10ex6 cells/mouse) or C.alb-S (CP620) (diabetic: 1.5x10ex6 cells/mouse; non-diabetic: $6.9 \times 10 \mathrm{ex} 6 \mathrm{cells} / \mathrm{mouse}$ ) and $24 \mathrm{~h}$ later, given $5 \mathrm{mg} / \mathrm{kg} \mathrm{AmBi}$ or $5 \%$ dextrose in water (D5W) intravenously for 6 days or Flu $40 \mathrm{mg} / \mathrm{kg}$ PO 2X/day for 6 days. Tissues (kidneys, liver, spleen) were collected ( $n=3-6 /$ group) on $d 4$ (diabetic) and $d 7$ (nondiabetic) post-challenge, homogenized and plated on Sabouraud's agar to determine fungal burden (CFU/g). Other mice ( $n=6-10 / g p$ ) were monitored for morbidity to d28. Results: With a C.alb-R infection, $A m B i$ treated, non-diabetic and diabetic mice, had significantly better survival than Flu or D5W treated mice (AmBi 83$100 \%$ survival, Flu $0-30 \%$ survival, D5W $(0-20 \%$ survival) ( $\leq \leq 0.01)$. Significant decreases in CFU/g kidneys were observed with $\mathrm{AmBi}$ treatment versus D5W or Flu in diabetic and non-diabetic mice ( $p \leq$ 0.04); no fungi were recovered in livers or spleens of $A m B i$ treated mice ( $p \leq 0.04$ ). In comparison, with C.alb-S infection, AmBi or Flu treated, non-diabetic mice had $100 \%$ and $80 \%$ survival, respectively, but in diabetic mice, survival was $100 \%$ for $\mathrm{AmBi}$ and only $16 \%$ for Flu even though this was a Flu sensitive strain $(p=0.0021)$. D5W yielded $0 \%$ (non-diabetic) and $14 \%$ (diabetic) survival. The fungal burden in the livers and spleens was significantly lower with $\mathrm{AmBi}$ treatment versus Flu or D5W, with AmBi treatment reducing the liver and spleen fungal burdens to undetectable levels. In the kidneys, the fungal burden in non-diabetic mice was significantly lower for $\mathrm{AmBi}$ versus Flu or D5W, but there was no significant difference in kidney fungal burden in diabetic mice given $\mathrm{AmBi}$ or Flu. Conclusions: In non-diabetic mice, $\mathrm{AmBi}$ and Flu were effective in treating systemic candidiasis caused by a $C$. albicans azole sensitive strain, while AmBi was effective in treating non-diabetic and diabetic mice infected with a $C$. albicans azole sensitive or resistant strain. Notably, in diabetic mice challenged with a $C$. albicans azole sensitive strain, Flu was associated with poor survival and elevated tissue fungal burden, indicating that the diabetic condition enhanced susceptibility to systemic candidiasis whether it was caused by a $C$. albicans azole sensitive or resistant strain.
\end{abstract}

\section{INTRODUCTION}

Diabetes is a chronic metabolic disorder due to improper insulin utilization or insufficient insulin production by the pancreatic $\beta$-cells, which eventually leads to hyperglycemia and long-term complications ${ }^{1}$. Obesity is a strong risk factor of type 2 diabetes. The excess accumulation of fat contributes to chronic low-grade inflammation, which together with hyperglycemia compromise the blood circulation, immune responses ${ }^{2,3}$, and metabolic pathways ${ }^{4}$ leading to increased susceptibility to infection such as candidiasis ${ }^{5}$.

Fluconazole (Flu), has been used as first line therapy for localized and systemic candidiasis ${ }^{6}$, but the incidence of azole resistance is rising ${ }^{7}$. Unlike azoles, the incidence of resistance to polyene, broad spectrum, antifungal drugs, such as liposomal amphotericin B (AmBisome ${ }^{\circledR}[\mathrm{AmBi}]$ ) remains minimal. This study was done to compare the efficacy of Flu versus $A m B i$ in diabetic vs. non-diabetic mice with systemic candidiasis when it is caused by a C. albicans azole sensitive(C.alb-S) or azole resistant $(C . a l b-R)$ strain

RESULTS

Fig 1-2. AmB and Flu treatment against azole sensitive $C$. albicans

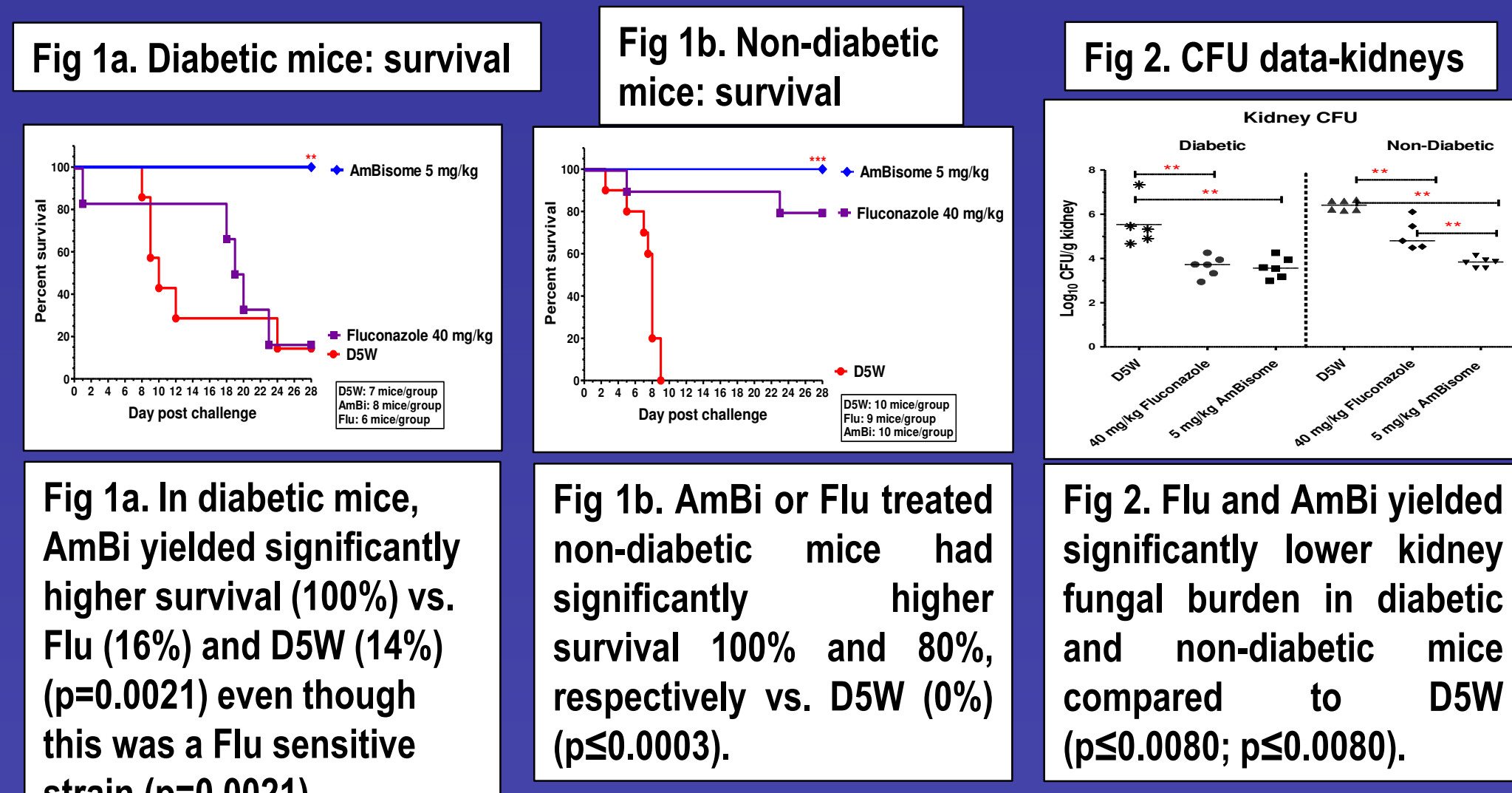
strain $(p=0.0021)$.

Fig 3-4. AmBi and Flu treatment against azole resistant $C$. albicans

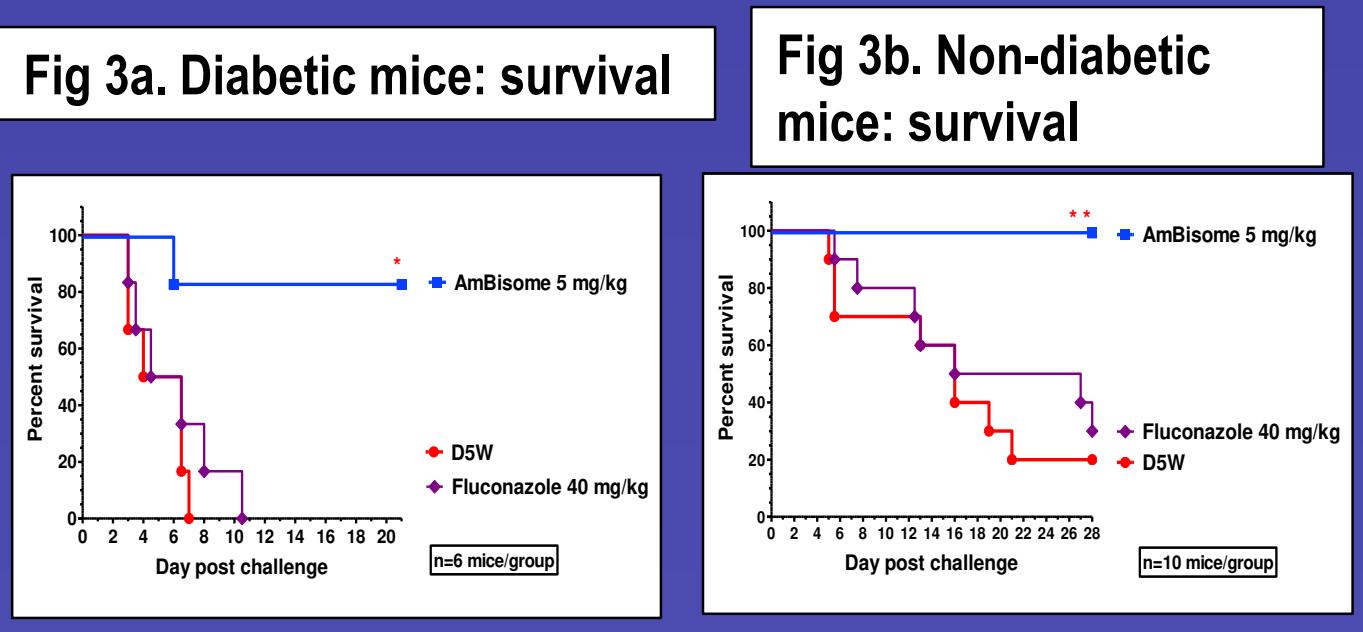

Fig3 a,b. *AmBi treated diabetic and non-diabetic mice had significantly higher survival $(83-100 \%)$ vs. Flu $(0-30 \%)$ and D5W $(0-20 \%)(p \leq 0.01)$

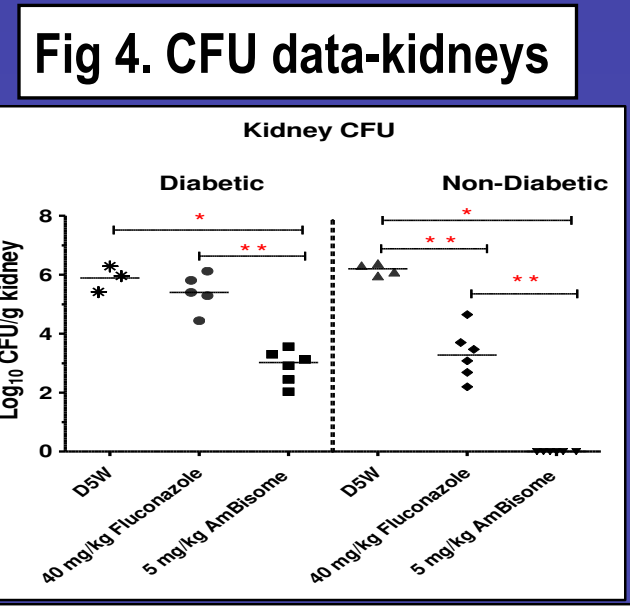

Fig4. ${ }^{*} \mathrm{AmBi}$ significantly decreased the kidney fungal burden in diabetic and nondiabetic mice vs Flu and D5W $(p \leq 0.02)$

\section{METHODS}

- ICR male mice (3-4 weeks old) were induced by high fat diet $(60 \% \mathrm{kCal} / \mathrm{fat})$ for 3-4 weeks - Diabetes induction: nicotinamide $(60 \mathrm{mg} / \mathrm{kg}$ ) and 15 minutes later streptozotocin (100 $\mathrm{mg} / \mathrm{kg}$ ) treatment.

The weights and blood glucose levels were monitored $2 x /$ week until the mean blood glucose levels were $\geq 200 \mathrm{mg} / \mathrm{dL}$.

Diabetic mice ( $n=9-14 / g p)$ and non-diabetic mice ( $n=14-16 / g p)$ were challenged intravenously (IV) with C.alb-R (ATCC 62342, Flu MIC >500ug $/ \mathrm{ml}, \mathrm{AmBi} 1.25 \mathrm{ug} / \mathrm{ml}$ ) (diabetic: $3.5 \times 10^{6} \mathrm{cells} / \mathrm{mouse}$; non-diabetic: $1.4 \times 10^{6} \mathrm{cells} / \mathrm{mouse}$ ) or C.alb-S (CP 620 , Flu MIC=2ug $/ \mathrm{ml}$ AmBi=0.625ug/ml) (diabetic: $1.5 \times 10^{6}$ cells $/$ mouse; non-diabetic: $6.9 \times 10^{6}$ cells $/$ mouse).

Treatments: $5 \mathrm{mg} / \mathrm{kg} \mathrm{AmBi}$ or $5 \%$ dextrose in water (D5W), IV or Flu $40 \mathrm{mg} / \mathrm{kg}$ PO 2X/day for $6 \mathrm{~d}$.

Tissues (kidneys, liver, spleen) were collected ( $n=3-6 / g r o u p)$ on $\mathrm{d} 4$ (diabetic) and $\mathrm{d} 7$ (nondiabetic) post-challenge, to determine fungal burden (CFU/g). Other mice ( $n=6-10 / g p)$ were monitored for morbidity and survival to $\mathrm{d} 28$.

\section{CONCLUSIONS}

- AmBisome was effective in treating systemic candidiasis in diabetic and non-diabetic mice caused by $C . a l b-S$ or $C . a l b-R$ strains based on increased survival and reduced fungal burden.

- Fluconazole was not effective for treating systemic candidiasis in either diabetic or non-diabetic mice when it was caused by C.alb- $R$.

- Fluconazole was effective in treating systemic candidiasis caused by C.alb-S in non-diabetic, but not diabetic mice.

- Since diabetes reduces the efficacy of Fluconazole but not AmBisome for treatment of systemic candidiasis, it is possible that this observation may be true for other fungal infections.

\section{ACKNOWLEDGMENTS}

Support Provided by research grants from: 